\title{
Bandgap Tunability in a One-Dimensional System
}

\author{
Payal Wadhwa ${ }^{1}$, Shailesh Kumar ${ }^{2,3}$, T.J. Dhilip Kumar ${ }^{4}$, Alok Shukla ${ }^{5}$ and Rakesh Kumar ${ }^{1, *}$ \\ 1 Department of Physics, Indian Institute of Technology Ropar, Nangal Road, Rupnagar, Punjab 140001, India; \\ payal.wadhwa@iitrpr.ac.in \\ 2 Manufacturing Flagship, CSIRO, Lindfield West, New South Wales 2070, Australia; Shailesh.Kumar@csiro.au \\ 3 School of Chemistry, Physics and Mechanical Engineering, Queensland University of Technology, Brisbane, \\ Queensland 4000, Australia \\ 4 Department of Chemistry, Indian Institute of Technology Ropar, Rupnagar, Punjab 140001, India; \\ dhilip@iitrpr.ac.in \\ 5 Department of Physics, Indian Institute of Technology Bombay, Powai, Mumbai 400076, India; \\ shukla@phy.iitb.ac.in \\ * Correspondence: rakesh@iitrpr.ac.in
}

Received: 20 September 2018; Accepted: 20 October 2018; Published: 22 October 2018

\begin{abstract}
The ability to tune the gaps of direct bandgap materials has tremendous potential for applications in the fields of LEDs and solar cells. However, lack of reproducibility of bandgaps due to quantum confinement observed in experiments on reduced dimensional materials, severely affects tunability of their bandgaps. In this article, we report broad theoretical investigations of direct bandgap one-dimensional functionalized isomeric system using their periodic potential profile, where bandgap tunability is demonstrated simply by modifying the potential profile by changing the position of the functional group in a periodic supercell. We found that bandgap in one-dimensional isomeric systems having the same functional group depends upon the width and depth of the deepest potential well at global minimum and derived correlations are verified for known synthetic as well as natural polymers (biological and organic), and also for other one-dimensional direct bandgap systems. This insight would greatly help experimentalists in designing new isomeric systems with different bandgap values for polymers and one-dimensional inorganic systems for possible applications in LEDs and solar cells.
\end{abstract}

Keywords: density functional theory; bandgap; polymers; nanoribbons; one-dimensional systems

\section{Introduction}

One-dimensional materials are in focus amongst the current research areas for their remarkable physical properties arising as a consequence of the reduced dimensionality. However, lack of control over reproducibility of bandgap values in one-dimensional materials [1-3] is one of the challenges for its electronic applications like LEDs and LASER diodes, as it affects their bandgap tunability. Several theoretical studies have been reported to tune the bandgap of one-dimensional materials using various methods like strain, functionalization at the edges, doping, etc. [4-6], however, the methods lack in control over the bandgap values. Since, bandgap is one of the most important factors while selecting a material for an electronic application; therefore, different direct bandgap materials have been explored for LEDs and LASER applications, e.g., Aluminium gallium nitride (AlGaN) for ultraviolet LEDs (below $400 \mathrm{~nm}$ ), while Aluminium gallium arsenide (AlGaAs) for infrared LEDs (above $760 \mathrm{~nm}$ ). Therefore, it would be of great interest if bandgap of a given material can be tuned, and this quest has been extended to polymers. Several experimental and theoretical studies on bandgap in polymers [7-13] have been reported aimed at their applications in organic LEDs [14-16] and solar cells [9,17-22]. However, different values of bandgap noticed in experiments on isomeric polymers [23-25] and also in theoretical studies $[12,25-27]$ suggest that bandgaps may be tuned in one-dimensional system, if the 
underlying physics is understood. The fact that the work so far reported on bandgaps in isomeric polymers having the same functional group is still inconclusive [25-27], motivated us to investigate and define the driving elements of different bandgaps seen in the isomeric systems. Once we understand the mechanism behind this, we may be in a position to tune the bandgaps of such materials as per our requirements. In this work, investigations are carried out on one-dimensional isomeric polymers (synthetic and natural), and nanoribbons having the same functional groups. Since polymers are large chain of monomers, Therefore, they are considered as one-dimensional periodic systems for band structure calculations [28-32].

\section{Results and Discussions}

For investigating and defining the driving element of different bandgaps in isomeric systems, an example of low bandgap synthetic polymer, polydithienyl naphthodithiophenes (DThNDT) $\left(\mathrm{C}_{20} \mathrm{H}_{8} \mathrm{~S}_{4}\right)_{n}$ is considered. It exists in two isomeric forms, poly(anti-DThNDT) and poly(syn-DThNDT) [25] having periodic unit cell of length $14.681 \AA$, and $13.113 \AA$, respectively (see Figure 1a). Cut-off energy of $500 \mathrm{eV}$ is used for band structure calculations. Ground state energy calculated per atom for poly(anti-DThNDT) and poly(syn-DThNDT) are $-7.034 \mathrm{eV}$ and $-7.003 \mathrm{eV}$, respectively, which are in close proximity of being isomers.

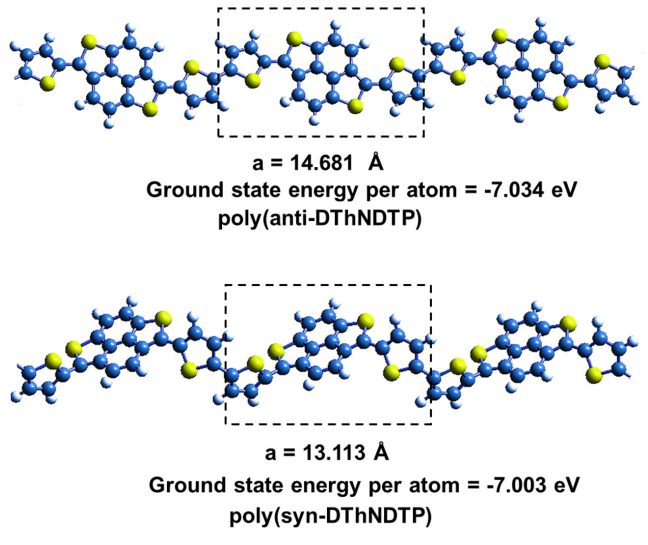

(a)

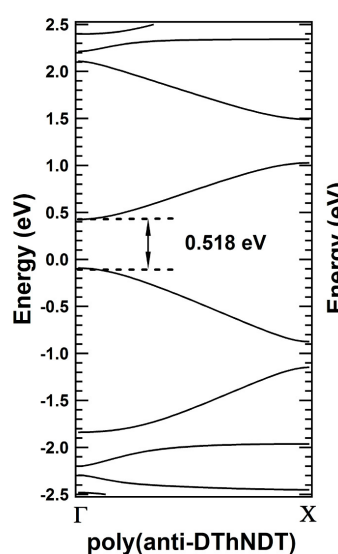

poly(anti-DThNDT)

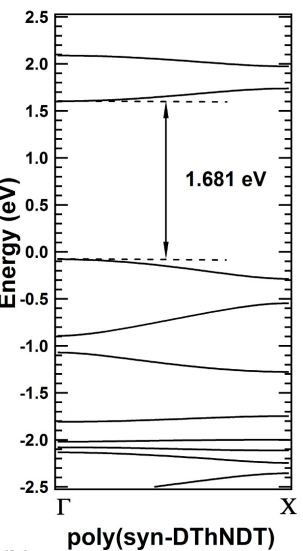

(b)

Figure 1. (color online) (a) Unit cells for poly(anti-DThNDT) and poly(syn-DThNDT) are represented in the dotted boxes. Blue, yellow, and white spheres represent carbon, sulfur, and hydrogen atoms, respectively; (b) Band structure plots corresponding to poly(anti-DThNDTP) and poly(syn-DThNDTP).

Given the one-dimensional nature of polymers, their band structures are plotted from $\Gamma$ to $X$ point (see Figure 1b). Direct bandgaps are observed at $\Gamma$ point for both the polymers of poly(anti-DThNDT) and poly(syn-DThNDT) with bandgaps of $0.518 \mathrm{eV}$ and $1.681 \mathrm{eV}$, respectively. Bandgap for poly(anti-DThNDT) is smaller than that of poly(syn-DThNDT), which is in agreement with the experimental report [25]. Band structures for these isomeric polymers are significantly different, even though they have same chemical formula, and practically the same ground state energy.

The polymers are one-dimensional systems with a repeating unit cell (monomer), Therefore, their bandgap may be related to their one-dimensional periodic potential profile similar to that of Kronig-Penney model. Since, potential is a scalar quantity, Therefore, average of potentials in the periodic direction of isomeric unit cell may be considered for comparative analysis of bandgap values. Average potential profile for poly(anti-DThNDT) and poly(syn-DThNDT) are plotted in the periodic direction as shown in Figure 2. 


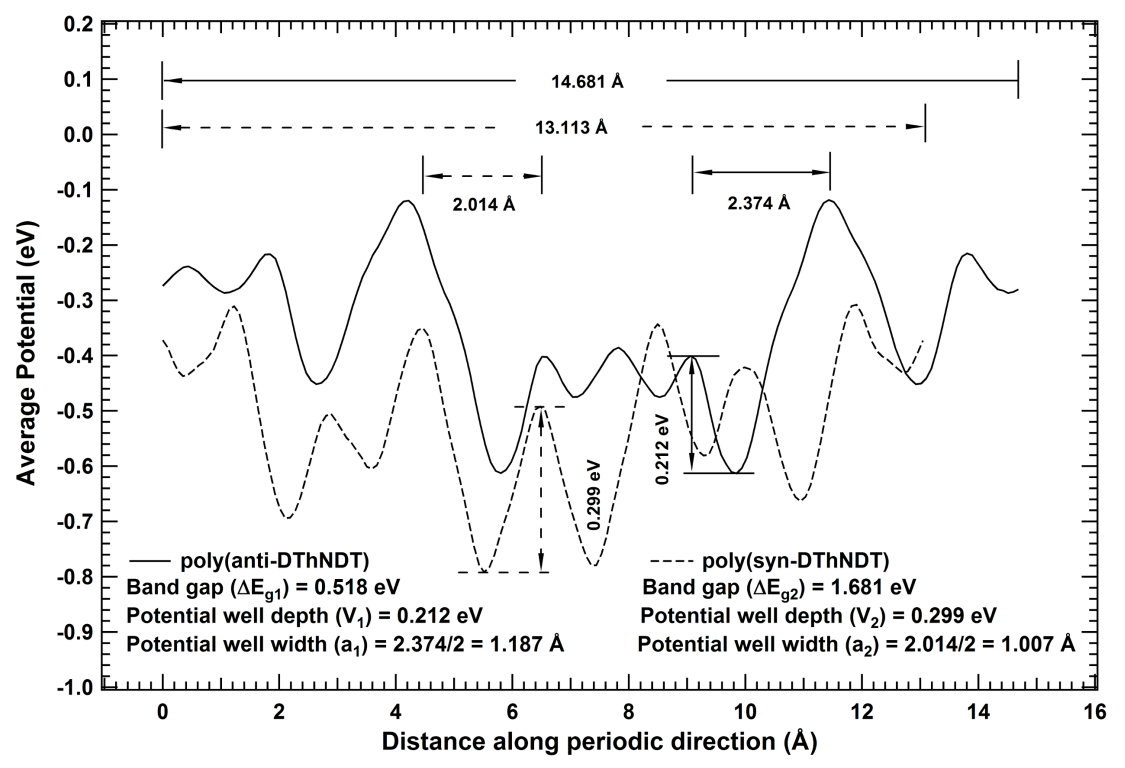

Figure 2. Average potential profile corresponding to the unit cell in the periodic direction of poly(antiDThNDT) and poly(syn-DThNDT) are denoted in solid and dotted lines, respectively.

The periodic average potential profiles of poly(anti-DThNDT) and poly(syn-DThNDT) are quite different to each other, and even to ideal rectangular potentials of the Kronig-Penney model [33]. Since a system prefers to stay in its ground state, the deepest potential well at global minimum in the periodic potential profiles are considered for comparative analysis of bandgap values [34] for isomeric systems. The global minimum for poly (anti-DThNDT) is located at $9.849 \AA$, enclosed between two crests (barriers) located at $9.058 \AA$ and $11.432 \AA$, while global minimum for poly(syn-DThNDT) is located at $5.517 \AA$, enclosed between two crests (barriers) located at $4.466 \AA$ and $6.480 \AA$. From Figure 2, it can be seen that shape of the potential wells in the potential profile looks like inverse Gaussians, consisting of both well and barrier width. Therefore, for simplifying the calculations, potential well is considered as square well potential of equal width for well and barrier (half of the distance between crests of the potential well). Since depth of the deepest potential well at global minimum ' $V_{0}$ ', and its corresponding width ' $a$ ' are finite and non-zero, distinct from the KP model (where $a \rightarrow 0$ and $V_{0} \rightarrow \infty$ for finite value of $V_{0} . a$ ) [33]. Hence, Schrödinger equation needs to be solved for the periodic square well potential of finite width and depth at global minimum to get first order bandgap, which may be correlated with the bandgap calculated using DFT. The energy eigenvalues corresponding to Schrödinger wave equation for an electron of mass ' $m$ ' and energy ' $E$ ' (where $E<V_{0}$ ) in a square well periodic potentials of finite depth ' $V_{0}$ ' and width ' $a$ ' can be obtained by solving the transcendental equation

$$
\frac{\beta^{2}-\alpha^{2}}{2 \alpha \beta} \sin (\alpha a) \sinh (\beta a)+\cos (\alpha a) \cosh (\beta a)=\cos (k .2 a)
$$

where,

$$
\beta=\sqrt{\frac{2 m V_{0}}{\hbar^{2}}-\alpha^{2}}, \frac{\hbar^{2} \alpha^{2}}{2 m}=E+V_{0}>0
$$

and,

$$
\frac{\hbar^{2} \beta^{2}}{2 m}=-E<0
$$


For isomeric polymers of polydithienyl naphthodithiophenes (DThNDT) $\left(\mathrm{C}_{20} \mathrm{H}_{8} S_{4}\right)_{n}$, width and depth of the deepest potential well at global minimum for poly(anti-DThNDT) are $1.187 \AA$ and $0.212 \mathrm{eV}$, respectively, while for poly(syn-DThNDT) are $1.007 \AA$ and $0.299 \mathrm{eV}$, respectively (Figure 2). Using these values in Equation (1), it is found that poly(syn-DThNDT) has larger bandgap than that of poly(anti-DThNDT), which is in agreement with bandgap values calculated using DFT, and experimental reports [25]. Therefore, it is concluded that bandgap of one-dimensional isomeric systems may be correlated with depth and width of the potential well at global minimum in the periodic average potential profile.

In order to find out how bandgap of one-dimensional isomeric systems may be correlated with their deepest potential well at global minimum in the periodic direction; a general correlation needs to be formulated and establish its validity for other isomeric systems. Since isomeric systems usually would have different dimensions ( $V_{0}$ and $a$ ) of the deepest potential well at global minimum, Therefore, the transcendental Equation (1) needs to be solved for bound states of different ' $V_{0} \cdot a^{\prime}$ varying both ' $V_{0}$ ' and ' $a$ '. In fact for bound states $\left(E<V_{0}\right)$, depth of potential well ' $V_{0}$ ' should be greater than a critical value for a given width ' $a$ ', which we have considered in the calculations; for example, the critical value of depth calculated for potential well is $19.04 \mathrm{eV}$ for a width of $1.4 \AA$, while critical depth turns out to be $16.59 \mathrm{eV}$ for a width of $1.5 \AA$. Bandgap for bound state periodic square well potential obtained from energy-momentum dispersion relations on solving the transcendental Equation (1), for different ' $V_{0} \cdot a^{\prime}$ are plotted in Figure 3.

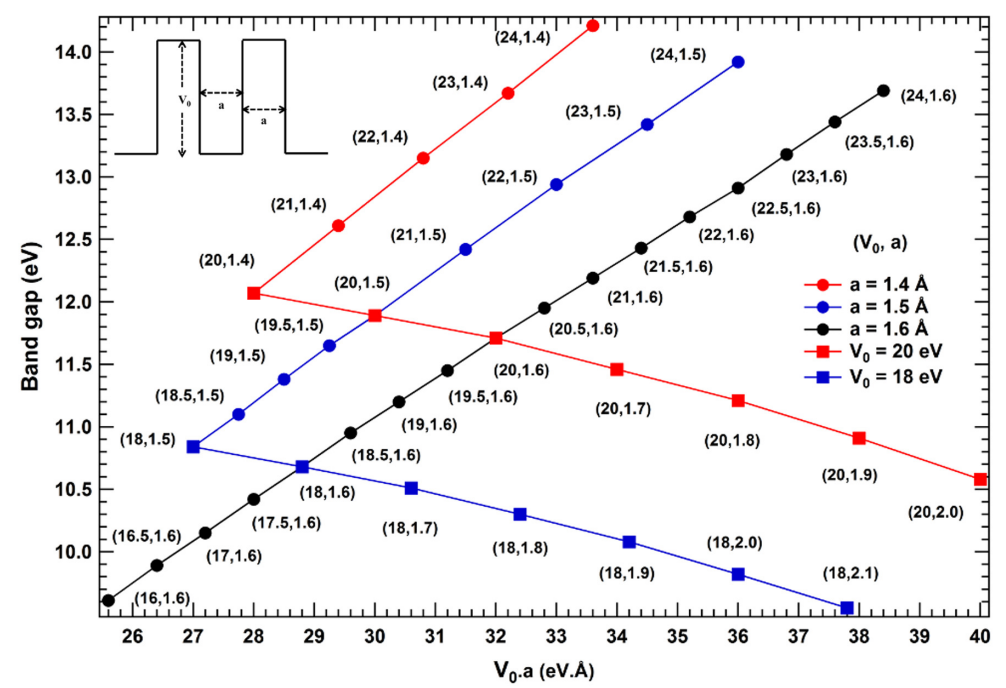

Figure 3. (color online) First order bandgap plot as a function of ' $V_{0} \cdot a^{\prime}$ for different values of ' $V_{0}$ ' and ' $a$ ' for the bound systems corresponding to a square well potential represented in the inset.

On comparing the bandgap values of two isomeric systems with their corresponding width (' $a$ ') and depth (' $V_{0}$ ') of potential well e.g., $\left(\triangle E_{g 1}, V_{1}, a_{1}\right)$ and $\left(\triangle E_{g 2}, V_{2}, a_{2}\right)$ for different product of ' $V_{0} \cdot a^{\prime}$, following correlations are derived

Case I $\left(a_{1}=a_{2}, V_{1}=V_{2}\right)$ then $\triangle E_{g 1}=\triangle E_{g 2}$

Case II $\left(a_{1}=a_{2}, V_{1}>V_{2}\right)$ then $\triangle E_{g 1}>\triangle E_{g 2}$

Case III $\left(a_{1}>a_{2}, V_{1}=V_{2}\right)$ then $\triangle E_{g 2}>\triangle E_{g 1}$

Case IV $\left(a_{1}>a_{2}, V_{2}>V_{1}\right)$ then $\triangle E_{g 2}>\triangle E_{g 1}$

Case V $\left(a_{1}>a_{2}, V_{1}>V_{2}\right)$

In this case, sign of slopes for bandgap as a function of ' $V_{0} \cdot a^{\prime}$ may change on changing ' $V_{0}$ ' and ' $a$ ' w.r.t. a reference point, Therefore, bandgap correlation can be predicted only on solving Equation (1) for corresponding ' $V_{0}$ ' and ' $a$ '. 
For isomeric polymers of polydithienyl naphthodithiophenes (DThNDT) $\left(\mathrm{C}_{20} \mathrm{H}_{8} \mathrm{~S}_{4}\right)_{n}$, the width and depth of the deepest potential well at global minimum in the periodic potential profile for poly(anti-DThNDT) are $1.187 \AA$ (say ' $a_{1}{ }^{\prime}$ ) and $0.212 \mathrm{eV}$ (say $V_{1}$ ), respectively, while for poly(syn-DThNDT) are $1.007 \AA$ (say ' $a_{2}$ ') and $0.299 \mathrm{eV}$ (say $V_{2}$ ), respectively (see Figure 2). Since $a_{1}>a_{2}$ and $V_{2}>V_{1}$, Therefore, according to correlations of Case IV, poly(syn-DThNDT) should have larger bandgap than poly(anti-DThNDT); which agrees with our band structure calculations using DFT, and other experimental reports [25]. The agreement of derived correlation with theoretical and experimental results establishes its validity.

To verify it further, the investigation is extended to other isomeric synthetic polymer polydialkylterthiophenes $\left(\mathrm{C}_{36} \mathrm{H}_{54} \mathrm{~S}_{3}\right)_{n}$ (see Supplementary Material Figure S1) and natural polymers (biopolymers and organic polymers) (see Supplementary Material Figure S2 and S3), bandgaps are found to be correlated with the dimension of the potential well at a global minimum as per the derived correlations. The investigations have been extended to natural polymers for extensive validity of the correlations, even though they are insulating and of little importance to electronic applications.

On the basis of theoretical analyses, it is established that bandgaps of isomeric systems are correlated with width and depth of the deepest potential well at global minimum in their periodic potential profile. From derived correlations, it may be predicted that bandgap of one-dimensional periodic system may be tuned, if width and depth of the deepest potential well in the periodic potential profile is altered on changing the position of functional group in the periodic unit cell.

To establish the concept of bandgap tunability in one-dimensional systems, the investigation is extended to theoretical GNRs (same molecular formula for the unit cells) having the same functional group in the periodic unit cell but of different arrangements. Zigzag GNRs (ZGNRs) of the same width functionalized at the edges with oxygen atoms in two typical ways (say Config. I and Config. II as shown in Figure 4a) are considered for calculations. $s p^{2}$ and $s p^{3}$ hybridized carbon atoms are considered at edges for visible distinction of isomeric change in the periodic unit cell (7.378 $\AA$ ) of zigzag GNRs. Typical edge configurations of ZGNRs $\left(N_{z}=7\right)$ are shown in Figure 4a. Periodic average potential profiles corresponding to Config. I and Config. II are plotted in Figure $4 \mathrm{~b}$. Even though their average potential profiles look different, their potential profiles superpose on each other on relative shifting in the periodic direction. The width and depth of potential well at global minimum for Config. I and Config. II are exactly same $0.614 \AA\left(a_{1}=a_{2}\right)$ and $0.648 \mathrm{eV}\left(V_{1}=V_{2}\right)$. Since $a_{1}=a_{2}$ and $V_{1}=V_{2}$, Therefore, according to the derived correlations (Case I), bandgap of both the configurations of ZGNRs should be equal.

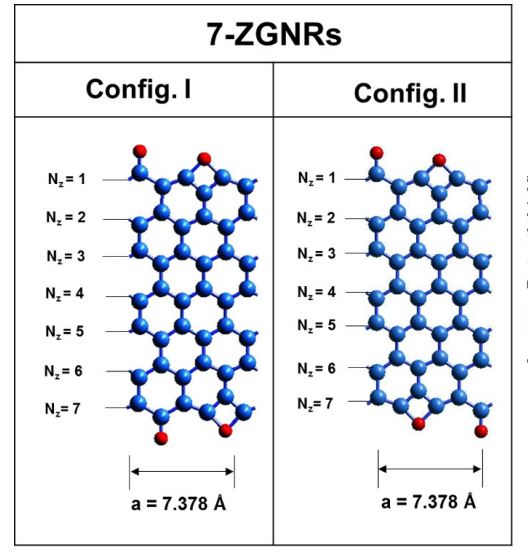

(a)

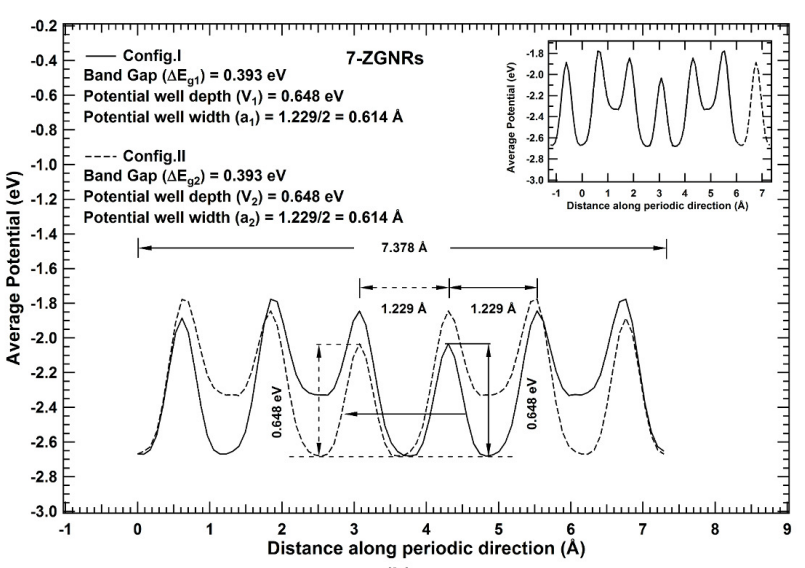

(b)

Figure 4. (color online) (a) Unit cells corresponding to two configurations Config. I and Config. II of 7-ZGNRs, where blue and red spheres represent carbon and oxygen atoms, respectively. (b) Average potential profile of the unit cell for Config. I and Config. II in the periodic direction of 7-ZGNRs are denoted with solid and dotted curve, respectively. Inset shows the overlap of potential profiles on relative shifting along the periodic direction. 
To check the validity of the correlations, band structure calculations for 7-ZGNRs are performed with cut-off energy of $450 \mathrm{eV}$. Band structures are plotted from $\Gamma$ to X point for $N_{z}=7$ (Figure 5). Direct bandgap of $0.393 \mathrm{eV}$ is observed at $\Gamma$ point for both the configurations. It is found that bandgap values of ZGNRs for Config. I and Config. II are same, even ground state energy per atom of the unit cell calculated for both the configurations are same, $-8.762 \mathrm{eV}$. On further analyses of potential profiles of ZGNRs, the same correlations are found to be held for other odd ZGNRs $\left(N_{z}=3\right.$ to 17), which are verified with the band structure calculations using DFT.
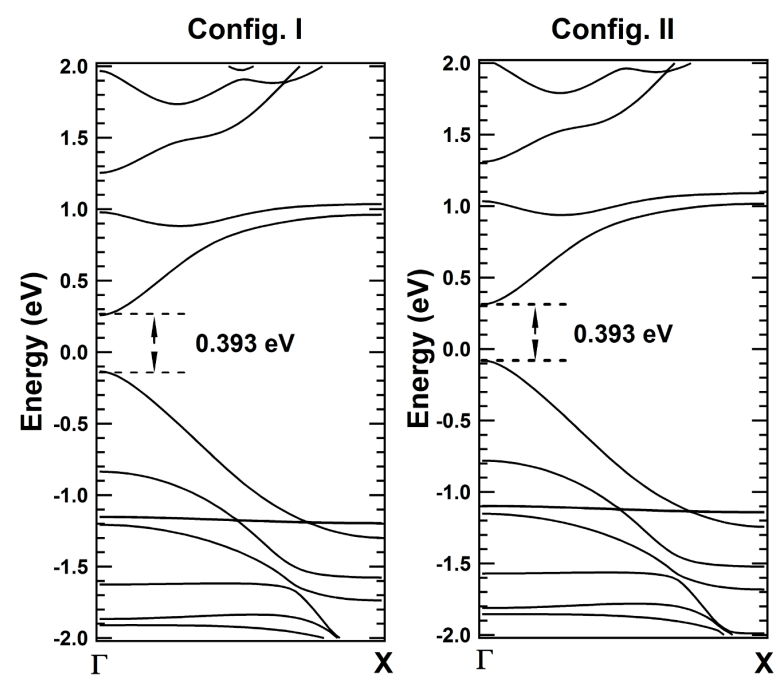

Figure 5. Band structure plots corresponding to Config. I and Config. II for $N_{z}=7$.

Further, we have considered even $N_{z}$-ZGNRs $\left(N_{z}=8\right)$. The unit cells corresponding to Config. I and Config. II are shown in Figure 6 a for $N_{Z}=8$. The lattice parameters in periodic direction for both the configurations are equal to $7.378 \AA$ A. The ground state energy per atom for Config. I and Config. II are $-8.818 \mathrm{eV}$ and $-8.817 \mathrm{eV}$, respectively, which are practically the same.

Average potential profiles in the periodic direction of unit cell corresponding to Config. I and Config. II are plotted in Figure $6 \mathrm{~b}$. The width and depth of potential well at global minimum for Config. I are $0.614 \AA\left({ }^{\prime} a_{1}{ }^{\prime}\right)$ and $1.091 \mathrm{eV}\left(V_{1}\right)$, respectively, while for Config. II are $0.614 \AA\left({ }^{\prime} a_{2}{ }^{\prime}\right)$ and $0.721 \mathrm{eV}\left(V_{2}\right)$, respectively. Since $a_{1}=a_{2}$ and $V_{1}>V_{2}$, Therefore, from the proposed theory, Config. I should have higher bandgap than that of Config. II (Case II). From band structure calculations, direct bandgaps of $0.792 \mathrm{eV}$ and $0.342 \mathrm{eV}$ are observed at $\Gamma$ point for Config. I and Config. II, respectively (Figure 7). Thus, bandgap of Config. I is significantly higher than that of Config. II, which is in agreement with derived correlation using potential profiles (Figure 6). On further analyses of potential profiles of even ZGNRs, the same correlations are found to be hold for other even ZGNRs $\left(N_{z}=10\right.$ to 18), which are verified with the band structure calculations using DFT. However, potential profiles of even $N_{z}$-ZGNRs for $N_{z}<6$ are found to fall under Case V (see Supplementary Material Figure S4). Thus, it justifies tunability of a bandgap value in direct bandgap one-dimensional systems. 


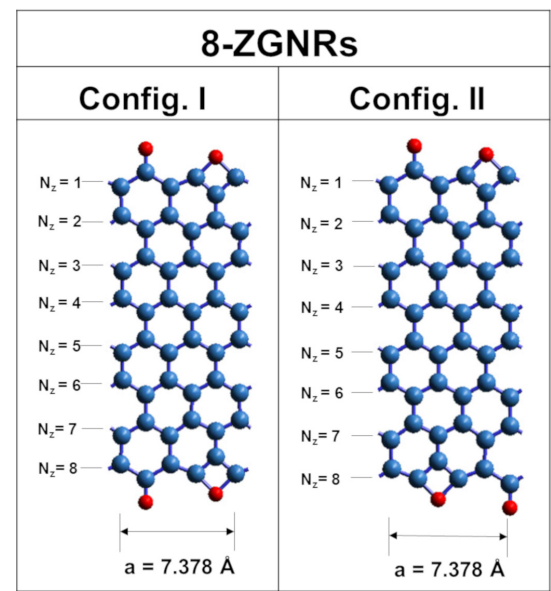

(a)

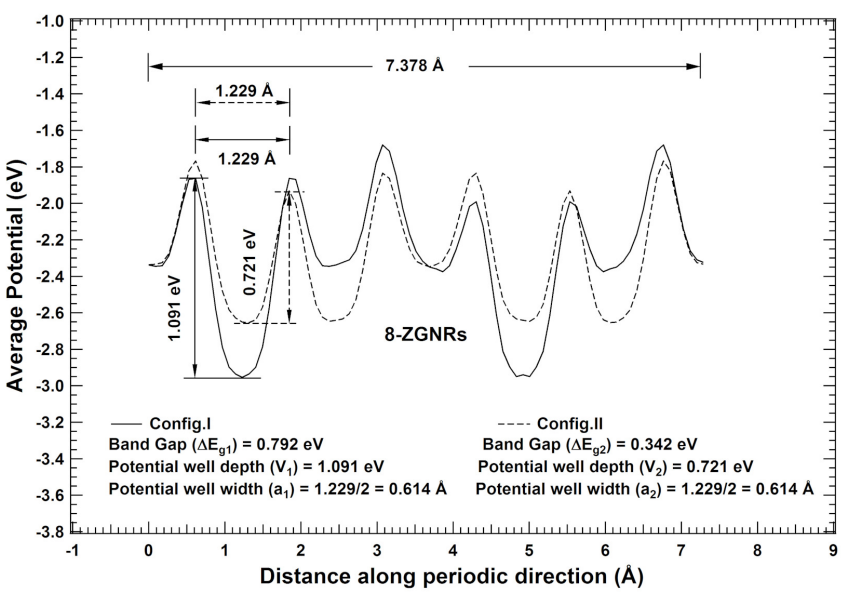

(b)

Figure 6. (color online) (a) Unit cells corresponding to two different configurations Config. I and Config. II of even ZGNRs corresponding to $N_{z}=8$, where blue and red spheres represent carbon and oxygen atoms, respectively; (b) their corresponding potential profiles.

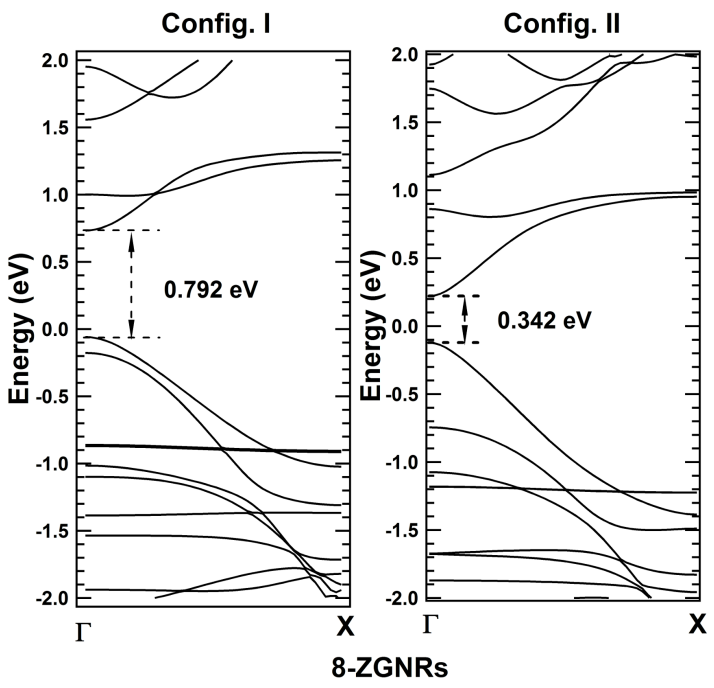

Figure 7. Band structure plots corresponding to Config. I and Config. II for $N_{z}=8$

\section{Computational Details}

Band structure calculations are performed using Density Functional Theory (DFT) as implemented in Vienna ab initio simulation package (VASP) [35]. Generalized gradient approximation (GGA) [36] is used for exchange-correlation of electron-electron interactions as implemented in projected augmented wave (PAW) formalism [37]. Further, a vacuum layer of at least $\approx 15 \AA$ is used to avoid interlayer interactions. The system is relaxed until a force on each atom in the unit cell is less than $0.001 \mathrm{eV} . \AA^{-1}$. k-mesh of size $25 \times 1 \times 1$ is used in Monkhorst-Pack formalism for momentum space sampling.

\section{Conclusions}

On the basis of theoretical analyses of one-dimensional systems having the same functional group in the periodic unit cell, but of different arrangements, it is observed that:

- Bandgaps of one-dimensional systems are correlated to the depth and width of potential well at global minimum in the periodic potential profile.

- The correlations derived between bandgap and dimension of periodic potential well at global minimum is verified for known isomeric systems of synthetic as well as natural polymers 
(biological and organic), and bandgap tunability is also established for one-dimensional nanoribbons.

Finally, it is concluded that bandgap of one-dimensional system can be tuned by changing the position of functional group in the periodic unit cell of the same material, which may be used for designing materials of different bandgap values for LEDs applications and effectively harvesting energy in solar cells; and insight may be extended to understand the different physical properties of isomers of biopolymers such as proteins.

Supplementary Materials: The following are available online at http:/ / www.mdpi.com/2410-3896/3/4/34/s1, Figure S1: Verification of the proposed theory for different bandgap values in isomers of polydialkylterthiophenes, Figure S2: Verification of the proposed theory for different bandgap values in Cellulose and Starch, Figure S3: Verification of the proposed theory for different bandgap values in Kevlar and nomex, Figure S4: Verification of the proposed theory for different bandgap values in even zigzag graphene nanoribbons $\left(N_{z}=6\right)$ of different arrangements.

Author Contributions: R.K. formulated and supervised the study. P.W., S.K., T.J.D.K., A.S., and R.K. developed the model for work. P.W. performed all the computations. All authors discussed the results and co-wrote the paper and reviewed the manuscript.

Funding: This research received no external funding.

Acknowledgments: The author thanks IIT Ropar for providing the High Performance Supercomputing facility. We also thank S. Kaur for doing some preliminary calculations related to manuscript.

Conflicts of Interest: The authors declare no conflict of interest.

\section{Abbreviations}

The following abbreviations are used in this manuscript:

DFT Density functional theory

DThNDT polydithienyl naphthodithiophenes

ZGNRs Zigzag graphene nanoribbons

\section{References}

1. Han, M.Y.; Özyilmaz B.; Zhang Y.; Kim P. Energy Band-Gap Engineering of Graphene Nanoribbons. Phys. Rev. Lett. 2007, 98, 206805. [CrossRef] [PubMed]

2. Li, X.; Wang, X.; Zhang, L.; Lee, S.; Dai, H. Chemically Derived, Ultrasmooth Graphene Nanoribbon Semiconductors. Science 2008, 319, 1229-1232. [CrossRef] [PubMed]

3. Tseng, F.; Unluer, D.; Holcomb, K.; Stan, M.R.; Ghosh, A.W. Diluted chirality dependence in edge rough graphene nanoribbon field-effect transistors. Appl. Phys. Lett. 2009, 94, 223112. [CrossRef]

4. Yan, J.-A.; Xian, L.; Chou, M.Y. Structural and Electronic Properties of Oxidized Graphene. Phys. Rev. Lett. 2009, 103, 086802. [CrossRef] [PubMed]

5. Biel, B.; Blase, X.; Triozon, F.; Roche, S. Anomalous Doping Effects on Charge Transport in Graphene Nanoribbons. Phys. Rev. Lett. 2009, 102, 096803. [CrossRef] [PubMed]

6. Lu, Y.; Guo, J. band-gap of strained graphene nanoribbons. Nano Res. 2010, 3, 189. [CrossRef]

7. Patra, D.; Lee, J.; Lee, J.; Sredojevic, D.N.; White, A.J.P.; Bazzi, H.S.; Brothers, E.N.; Heeney, M.; Fang, L.; Yoon, M.H.; et al. Synthesis of low band-gap polymers based on pyrrolo[3,2-d: 4,5-d']bisthiazole (PBTz) and thienylenevinylene (TV) for organic thin-film transistors (OTFTs). J. Mater. Chem. C 2017, 5, 2247-2258. [CrossRef]

8. Hou, J.; Chen, H.-Y.; Zhang, S.; Li, G.; Yang, Y. Synthesis, Characterization, and Photovoltaic Properties of a Low band-gap Polymer Based on Silole-Containing Polythiophenes and 2,1,3-Benzothiadiazole. J. Am. Chem. Soc. 2008, 130, 16144-16145. [CrossRef] [PubMed]

9. Bundgaard, E.; Krebs, F.C. Low band-gap polymers for organic photovoltaics. Sol. Energy Mater. Sol. Cells 2007, 91, 954-985. [CrossRef]

10. Chen, W.-C.; Liu, C.-L.; Yen, C.-T.; Tsai, F.-C.; Tonzola, C.J.; Olson, N.; Jenekhe S.A. Theoretical and Experimental Characterization of Small band-gap Poly(3,4-ethylenedioxythiophene methine)s. Macromolecules 2004, 37, 5959. [CrossRef] 
11. Ajayaghosh, A. Donor-acceptor type low band-gap polymers: Polysquaraines and related systems. Chem. Soc. Rev. 2003, 32, 181-191. [CrossRef] [PubMed]

12. Hong, S.Y.; Lee, K.W. Small Band-Gap Polymers: Quantum-Chemical Study of Electronic Structures of Degenerate П-Conjugated Systems. Chem. Mater. 2000, 12, 155-160. [CrossRef]

13. Hong, S.Y. Zero Band-Gap Polymers: Quantum-Chemical Study of Electronic Structures of Degenerate П-Conjugated Systems. Chem. Mater. 2000, 12, 495-500. [CrossRef]

14. Hamid, S.H.; Usmani, A.M. Applications of Polymers in Led Devices. Polym.-Plast. Technol. Eng. 1984, 22, 155-176. [CrossRef]

15. Yu, D.X. Light-Emitting Devices with Conjugated Polymers. Int. J. Mol. Sci. 2011, 12, 1575-1594. [PubMed]

16. AlSalhi, M.S.; Alam, J.; Dass, L. A.; Raja, M. Recent Advances in Conjugated Polymers for Light Emitting Devices. Int. J. Mol. Sci. 2011, 12, 2036-2054. [CrossRef] [PubMed]

17. Wang, C.; Xu, X.; Zhang, W.; Bergqvist, J.; Xia, Y.; Meng, X.; Bini, K.; Ma, W.; Yartsev. A.; Vandewal, K.; et al. Low band-gap Polymer Solar Cells With Minimal Voltage Losses. Adv. Energy Mater. 2016, 6, 1600148. [CrossRef]

18. Mori, H.; Nonobe, H.; Nishihara, Y. Highly crystalline, low band-gap semiconducting polymers based on phenanthrodithiophene-benzothiadiazole for solar cells and transistors. Polym. Chem. 2016, 7, 1549. [CrossRef]

19. Yiu, A.T.; Beaujuge, P.M.; Lee, O.P.; Woo, C.H.; Toney, M.F.; Fréchet J.M.J. Side-Chain Tunability of Furan-Containing Low-Band-Gap Polymers Provides Control of Structural Order in Efficient Solar Cells. J. Am. Chem. Soc. 2012, 134, 2180-2185. [CrossRef] [PubMed]

20. Woo, C.H.; Beaujuge, P.M.; Holcombe, T.W.; Lee, O.P.; Fréchet, J.M.J. Incorporation of Furan into Low Band-Gap Polymers for Efficient Solar Cells. J. Am. Chem. Soc. 2010, 132, 15547-15549. [CrossRef] [PubMed]

21. Cheng, Y. J.; Yang, S. H.; Hsu, C.-S. Synthesis of Conjugated Polymers for Organic Solar Cell Applications. Chem. Rev. 2009, 109, 5868-5923. [CrossRef] [PubMed]

22. Hou, J.; Chen, H.-Y.; Zhang S.; Chen, R.I.; Yang, Y.; Wu, Y.; Li, G. Synthesis of a Low band-gap Polymer and Its Application in Highly Efficient Polymer Solar Cells. J. Am. Chem. Soc. 2009, 131, 15586-15587. [CrossRef] [PubMed]

23. Ying, L.; Huang, F.; Bazan, G.C. Regioregular narrow-bandgap-conjugated polymers for plastic electronics. Nat. Commun. 2017, 8, 14047. [CrossRef] [PubMed]

24. Huang, Y.; Xu, W.; Zhou, C.; Zhong, W.; Xie, R.; Gong, X.; Ying, L.; Huang, F.; Cao, Y. Synthesis of medium-bandgap П-Conjugated polymers based on isomers of 5-Alkylphenanthridin-6(5H)-one and 6-Alkoxylphenanthridine. J. Polym. Sci., Part A: Polym. Chem. 2016, 54, 2119-2127. [CrossRef]

25. Casado, J.; Joaquín Quirante, J.; Hernández, V.; López Navarrete, J.T.; Takimiya, K.; Otsubo, T. Raman and Computational Study of Two Dithienyl Naphthodithiophenes: Synthesis and Characterization of New Polymers Showing Low band-gap Optical and Electroactive Features. J. Phys. Chem. B 2004, 108, 7611-7619. [CrossRef]

26. Hong, S.Y.; Kim, S.C. Towards Designing Environmentally Stable Conjugated Polymers with very Small Band-Gaps. Bull. Korean Chem. Soc. 2003, 24, 1649-1654.

27. Kertesz, M. Structure and Electronic Structure of Low-Band-Gap Ladder Polymers. Macromolecules 1995, 28, 1475-1480. [CrossRef]

28. Fabish, T.J. Electronic structure of polymers. Crit. Rev. Solid State Mater. Sci. 1979, 8, 383-420. [CrossRef]

29. Salaneck, W. R.; Brédas, J. L. Electronic band structure of conjugated polymers. Synth. Met. 1994, 67, 15-22. [CrossRef]

30. Duke, B.J.; O'Leary, B. The band structure of polymers: Its calculation and interpretation. Part 2. Calculation. J. Chem. Educ. 1988, 65, 379. [CrossRef]

31. Duke, B.J.; O'Leary, B. The band structure of polymers: Its calculation and interpretation. Part 3. Interpretation. J. Chem. Educ. 1988, 65, 513. [CrossRef]

32. Duke, C.B.; Ford, W.K. The electronic structure of polymers: Relation to molecular architecture. Int. J. Quantum Chem. 1983, 24, 597-608. [CrossRef]

33. Kronig R. de L.; Penney W.G. Quantum Mechanics of Electrons in Crystal Lattices. Proc. R. Soc. Lond. Ser. A 1931, 130, 499-513. [CrossRef]

34. Deepika; Kumar S.; Shukla A.; Kumar R. 2016 Origin of multiple band-gap values in single width nanoribbons. Sci. Rep. 2016, 6, 36168. [CrossRef] [PubMed] 
35. Kresse G.; Furthmüller J. Efficiency of ab-initio total energy calculations for metals and semiconductors using a plane-wave basis set. Comput. Mater. Sci. 1996, 6, 15-50. [CrossRef]

36. Perdew J.P.; Chevary J.A.; Vosko S.H.; Jackson K.A.; Pederson M.R.; Singh D.J.; Fiolhais C. Atoms, molecules, solids, and surfaces: Applications of the generalized gradient approximation for exchange and correlation. Phys. Rev. B 1992, 46, 6671. [CrossRef]

37. Kresse G.; Joubert D. From ultrasoft pseudopotentials to the projector augmented-wave method. Phys. Rev. B 1999, 59, 1758. [CrossRef]

(C) 2018 by the authors. Licensee MDPI, Basel, Switzerland. This article is an open access article distributed under the terms and conditions of the Creative Commons Attribution (CC BY) license (http://creativecommons.org/licenses/by/4.0/). 